\title{
Experimental Study of the Effect of Photobiomodulation Therapy on the Regulation of the Healing Process of Chronic Wounds
}

\author{
Sergey B. Pavlov $\mathbb{D}^{1,2}$ Nataliia M. Babenko ${ }^{1,},{ }^{1,2}$ Marina V. Kumetchko $\mathbb{D}^{1}$ \\ Olga B. Litvinova $\left(\mathbb{C}^{1,2}\right.$ and Rostyslav N. Mikhaylusov $\mathbb{1}^{3}$ \\ ${ }^{1}$ Central Research Laboratory, Kharkiv Medical Academy of Postgraduate Education, Kharkiv 61176, Ukraine \\ ${ }^{2}$ Department of Human Anatomy and Physiology, H.S. Skovoroda Kharkiv National Pedagogical University, \\ Kharkiv 61168, Ukraine \\ ${ }^{3}$ Department of Endoscopy and Surgery, Kharkiv Medical Academy of Postgraduate Education, Kharkiv 61176, Ukraine
}

Correspondence should be addressed to Nataliia M. Babenko; natali_babenko@ukr.net

Received 23 April 2021; Revised 28 May 2021; Accepted 29 July 2021; Published 7 September 2021

Academic Editor: Rodrigo Alvaro Lopes-Martins

Copyright (c) 2021 Sergey B. Pavlov et al. This is an open access article distributed under the Creative Commons Attribution License, which permits unrestricted use, distribution, and reproduction in any medium, provided the original work is properly cited.

\begin{abstract}
Currently, wound treatment is an urgent task of medicine around the world. In the process of wound healing, various types of cells are involved under the control and regulation of cytokines and growth factors. Disruption of the synchronization process between the various types of cells and intercellular mediators involved in the restoration of tissue damage can lead to impaired healing and the development of chronic wounds. Photobiomodulation (PBM) therapy promotes platelet activation and aggregation, reduces inflammation and oxidative stress, and accelerates cell migration and proliferation. PBM also induces the production of the extracellular matrix and the release of key growth factors, thereby improving tissue regeneration and accelerating wound healing. The aim of our work was to study the effect of photobiomodulation therapy on the regulation of reparative processes in chronic wounds monitored by biomarkers and platelet aggregation activity. 54 Wistar rats were divided into three groups. Intact animals were not manipulated. In animals of the control and experimental groups, a chronic wound was simulated by reproducing the conditions of local hypoxia and microcirculation disorders. The wounds of the experimental group received PBM therapy. The device Lika-therapist M (Ukraine) was used in a continuous mode at a wavelength of $660 \mathrm{~nm}$, an output power of $10 \mathrm{~mW}$, and an energy density of $1 \mathrm{~J} / \mathrm{cm}^{2}$. The wounds of the animals in the control group were treated with sham. The animals were euthanized on days $3,7,14$, and 28 after the surgery ( 6 animals, each from the control and experimental groups). Measurements of the levels of interleukin-6 (IL-6), interleukin-10 (IL-10), tumor necrosis factor alpha (TNF- $\alpha$ ), the basic fibroblast growth factor (bFGF), and reactive oxygen species (ROS) were carried out by ELISA. Results revealed the multidirectional effect of PBM therapy on the expression of the studied biomarkers. The results of the histological examination indicated a positive effect of PBM therapy with the applied parameters on the repair processes of chronic wounds. We concluded that the use of PBM therapy made it possible to regulate disturbances in reparative processes by modulating ROS, cytokines, and platelet aggregation activity.
\end{abstract}

\section{Introduction}

The theoretical foundations of the possibility of creating a laser were laid by Albert Einstein, who first touched upon the possibility of stimulated radiation in a 1917 article. And in 1960, the revolutionary discovery of the laser by Theodore Maiman changed the world. Maiman presented his invention to the scientific community in an article in the journal Nature, which contained just under 300 words. Soon, a first application of low-intensity laser therapy for wound healing was demonstrated by the Hungarian doctor Endre Mester and his colleagues [1].

Today, photobiomodulation (PBM) therapy has found applications not only in the treatment of chronic wounds but also in the treatment of many diseases, including neurological and musculoskeletal [2]. Also, this noninvasive 
method is used to reduce pain [3]. Despite widespread studies of various aspects of the action of PBM therapy, the exact mechanisms of laser-tissue interaction are not fully understood. It also requires a deeper understanding of the parameters that determine the therapeutic results and effectiveness of this method of treatment.

Currently, the treatment of wounds, called the "silent epidemic" [4], continues to be an urgent task of medicine around the world. Wound healing is a multifaceted process driven by sequential but overlapping phases including hemostasis, inflammation, proliferation, and remodeling [5]. Acute wounds usually heal in a consistent and timely manner. Chronic wounds remain blocked in one of these stages (oftentimes the inflammatory phase). This process involves various types of cells under the control and regulation of cytokines and growth factors. Reactive oxygen species (ROS) are also important regulators of wound healing phases [6]. Disruption of the synchronization of dynamic interactions among these various types of cells and intercellular mediators can lead to impaired healing and the development of chronic wounds. PBM therapy promotes platelet activation and aggregation, reduces inflammation and oxidative stress, and accelerates cell migration and proliferation. It also induces the production of the extracellular matrix and the release of key growth factors, thereby improving tissue regeneration and accelerating wound healing $[7,8]$.

In the literature, there are numerous works that demonstrate positive effects of PBM therapy on individual phases of the healing process, mainly on the inflammation stage. Overall, it is of interest to study the regulation of the repair process of chronic wounds by intercellular mediators at all stages of healing when using PBM therapy. Animal models remain the best available alternative for studying the complex cellular and molecular interactions that occur during the wound healing process [9].

The aim of our work was to study the effect of photobiomodulation therapy on the regulation of reparative processes in chronic wounds using biomarkers (for example, interleukin-6 (IL-6), interleukin-10 (IL-10), tumor necrosis factor alpha (TNF- $\alpha$ ), the basic fibroblast growth factor (bFGF), and reactive oxygen species (ROS)) and platelet aggregation activity.

\section{Materials and Methods}

The work included 54 Wistar rats weighing $250 \pm 25 \mathrm{~g}$ at the age of 8-9 months. The study was approved by the Committee on Ethical Animal Care and Use of the Kharkiv Medical Academy of Postgraduate Education, Ukraine (Protocol No. 5 dated November 12, 2019). All animal handling procedures were performed in accordance with the rules and guidelines set by this Committee.

2.1. Study Design. All rats were randomly divided into three groups (see Figure 1). Intact (Int) animals were not manipulated. Wounds were induced in animals of the control (Con) and experimental (Exp) groups.

The literature mainly contains models of acute wounds or wounds in animals with systemic diseases, such as diabe- tes. We have chosen a model of a chronic wound that reproduces the conditions of local hypoxia and impaired microcirculation [10].

Each animal was anesthetized (zoletil $10 \mathrm{mg} / \mathrm{kg}$ body weight) and depilated. In the proximal part of the back, the skin was excised with surgical scissors to the superficial fascia in the form of a circle with a diameter of about $20 \mathrm{~mm}$. After that, a perpendicular loop-shaped fascial skin suture was applied along the edges of the wound. On the surface of the bottom of the formed wound, the superficial fascia was dissected by mutually perpendicular transverse and longitudinal cuts, which formed cells measuring $5 \times 5 \mathrm{~mm}$, which were then sutured with U-shaped sutures (see Figure 2).

The wounds of the experimental group received PBM therapy. The wounds of the animals in the control group were treated with sham.

For PBM therapy, the laser device Lika-therapist $M$ (Ukraine) was used once a day for 5 days, starting from 24 hours after the formation of the wound. The parameters of low-intensity laser radiation are shown in Table 1 . The beam of the laser tip was held perpendicular to the surface of the irradiated tissue. The distance between the extension handle and the wound was selected so as to illuminate the entire area of the wound. Taking into account the divergence of the diode laser beam $(0.5 \mathrm{rad} \pm 20 \%)$ and the aperture diameter of the laser device extension handle $(0.2 \mathrm{~cm})$ (recommended by Lika-therapist $\mathrm{M}$ ), the distance between the extension handle and the wound was $3.53 \mathrm{~cm}$.

The animals were taken out of the experiment on days 3 , 7,14 , and 28 after surgery (6 animals, each from the control and experimental groups). Animals were euthanized by inhalation of chloroform in a confined space.

Blood samples were taken by open cardiac puncture. Measurements of the levels of the studied indicators in the blood serum of animals were carried out by the method of enzyme-linked immunosorbent assay (ELISA). The method is based on a sandwich version of ELISA using mono- and polyclonal antibodies. ROS and bFGF concentrations were determined using ELISA kits (Elabscience Biotechnology Inc., USA) ROS (Catalog No: E-EL-H5436) and bFGF (Catalog No: E-EL-H0483) according to the manufacturers' instructions. The levels of IL-6, IL-10, and TNF- $\alpha$ were determined using the ELISA kits (Vector-Best, Russia) IL-6 (Catalog No: A-8768), IL-10 (Catalog No: A8774) and TNF- $\alpha$ (Catalog No: A-8756) following the manufacturers' instructions.

2.2. Determination of the Functional Activity of Platelets. The study of induced platelet aggregation was carried out in platelet-rich plasma. Sodium citrate $3.2 \%$ was used as an anticoagulant in a ratio of 9 parts blood to 1 part stabilizer. To obtain platelet-rich plasma, the blood was immediately centrifuged for $10-12 \mathrm{~min}$ at $1000 \mathrm{rpm}$. One part of the resulting plasma was taken into a plastic tube and stored in a thermostat at $37^{\circ} \mathrm{C}$ until analysis. The second part of the plasma was subjected to further centrifugation at $4000 \mathrm{rpm}$ for 20 minutes, thus obtaining platelet-poor plasma. This plasma was used to zero the optical density of the device and dilute the platelet-rich plasma. For the analysis, we took 


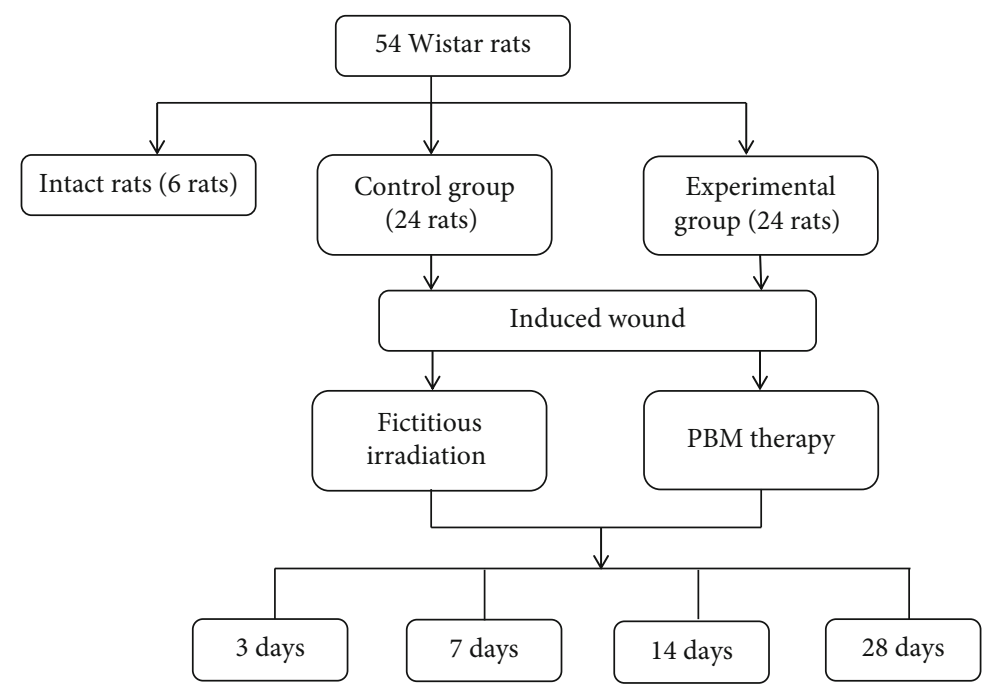

Figure 1: Study design.

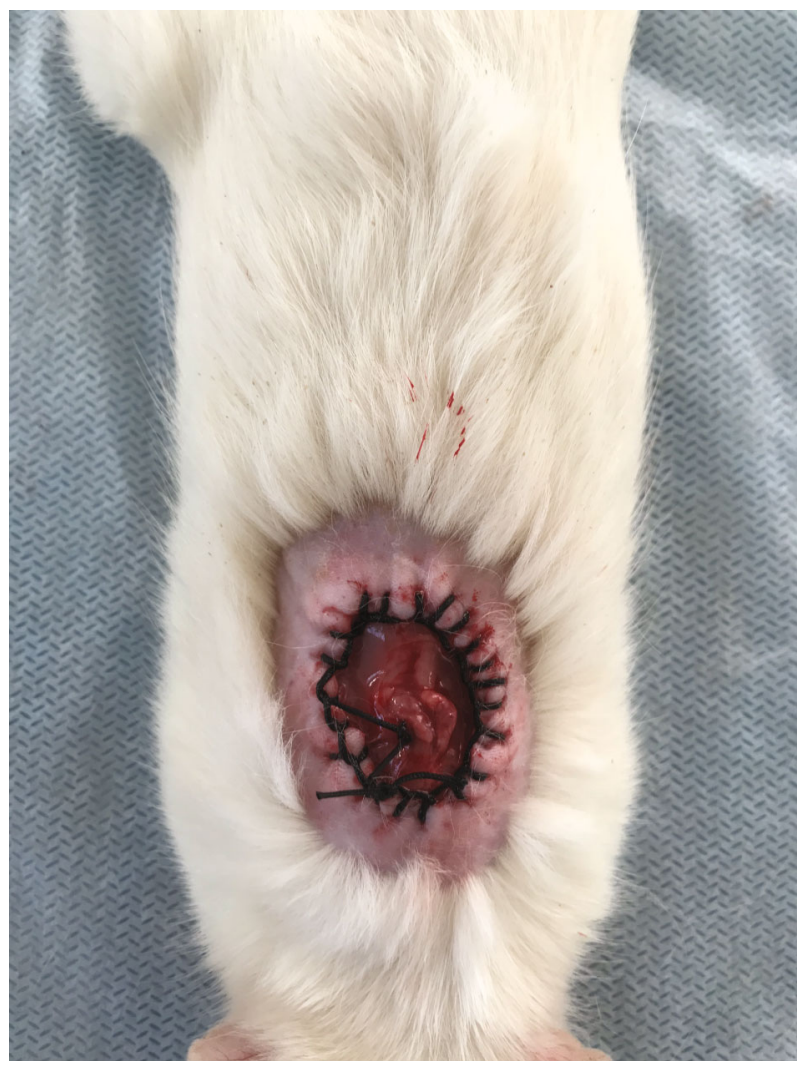

FIgURE 2: The induced injury.

plasma, keeping the concentration of platelets to 200-250 thousand/ $\mu \mathrm{l}$ or $43.5-52.5 \%$ of light transmission, relative to platelet-poor plasma. The study was carried out on a computerized analyzer of platelet aggregation SOLAR 2110 (Belarus). Adenosine diphosphate (ADP) (TekhnologiyaStandard, Russia) at concentrations of $2.5,5$, and $10 \mu \mathrm{mol} / 1$ was used to induce aggregation. An aggregatogram recording was carried out for 10 minutes at $37^{\circ} \mathrm{C}$. All studies were carried out in plasma obtained no later than 3 hours after blood sampling. The analysis of the aggregatograms included estimating the shape of the curves and determining the following: the degree of aggregation (the maximum level of plasma light transmission after the inductor was introduced), the rate of aggregation (measured over a 30-second segment at the most linear portion of the rise in the aggregation curve), and the aggregation time (the time to reach the maximum light transmission of the plasma).

2.3. Histological Analysis. After euthanasia of the animals, the wound area was surgically removed, fixed in $10 \%$ formalin, and embedded in paraffin. Subsequently, serial sections of 5-7 $\mu \mathrm{m}$ in size were obtained and stained with hematoxylin and eosin (H\&E). The preparations were analyzed and photographed using a Primostar microscope (Zeiss) and a microocular digital camera.

2.4. Statistical Analysis. Statistical processing of the results was performed using Statistica 6.0 (StatSoft, USA) statistical analysis package. The descriptive data were presented as $M$ $\pm \mathrm{SE}$, where $M$ is the arithmetic mean and SE is the standard error of the arithmetic mean. The significance of differences between groups (statistical significance) was determined using the nonparametric Kruskal-Wallis test for independent samples. The significance $p<0.05$ was considered statistically significant. Histograms were plotted by GraphPad Prism 9 (GraphPad Software, USA).

\section{Results}

Indicators of the degree, rate, and time of reaching the maximum rate of platelet aggregation at concentrations of the aggregation inducer $2.5,5$, and $10 \mu \mathrm{mol} / \mathrm{l} \mathrm{ADP}$ are shown in Table 2.

A significant decrease in the degree and rate of platelet aggregation was observed in rats with wounds at concentrations of an aggregation inducer of $2.5,5$, and $10 \mu \mathrm{mol} / 1$ on day 3 after surgery, compared to the control group (see Table 2). The time to reach the maximum rate of 
TABLE 1: Laser parameters.

\begin{tabular}{lcccccc}
\hline Wavelength $(\mathrm{nm})$ & Mode & Total energy & Power output $(\mathrm{mW})$ & Spot area $\left(\mathrm{cm}^{2}\right)$ & Time $(\mathrm{sec})$ & Energy density $\left(\mathrm{J} / \mathrm{cm}^{2}\right)$ \\
\hline 660 & Continuous & 3.14 & 10 & 3.14 & 314 & 1.0 \\
\hline
\end{tabular}

TABLE 2: . Indicators of functional activity of platelets in animals at various concentrations of adenosine diphosphate (ADP).

\begin{tabular}{|c|c|c|c|c|c|}
\hline \multirow{2}{*}{ Indicators } & \multicolumn{5}{|c|}{ Groups of animals } \\
\hline & Int & Con 3 days & Exp 3 days & Con 7 days & Exp 7 days \\
\hline $\begin{array}{l}\text { Aggregation degree (\%) } \\
2.5 \mu \mathrm{mol} / 1\end{array}$ & $27.26 \pm 2.44$ & $57.73 \pm 3.06$ & $20.95 \pm 5.89^{*}$ & $28.76 \pm 1.61$ & $23.77 \pm 5.64$ \\
\hline $\begin{array}{l}\text { Aggregation time (s) } \\
2.5 \mu \mathrm{mol} / 1\end{array}$ & $61.17 \pm 3.46$ & $102.33 \pm 7.22$ & $59.00 \pm 4.05^{*}$ & $61.40 \pm 2.86$ & $66.67 \pm 1.98$ \\
\hline $\begin{array}{l}\text { Aggregation rate for } 30 \mathrm{~s}(\% / \mathrm{min}) \\
2.5 \mu \mathrm{mol} / 1\end{array}$ & $56.77 \pm 2.05$ & $88.00 \pm 2.79$ & $54.10 \pm 6.70^{*}$ & $65.20 \pm 2.77$ & $52.07 \pm 8.15$ \\
\hline $\begin{array}{l}\text { Aggregation degree (\%) } \\
5 \mu \mathrm{mol} / 1\end{array}$ & $42.28 \pm 4.54$ & $72.50 \pm 0.44$ & $33.50 \pm 8.22^{*}$ & $45.34 \pm 2.16$ & $44.53 \pm 4.96$ \\
\hline $\begin{array}{l}\text { Aggregation time (s) } \\
5 \mu \mathrm{mol} / 1\end{array}$ & $108.17 \pm 7.56$ & $146.00 \pm 5.25$ & $106.33 \pm 6.45^{*}$ & $100.60 \pm 8.33$ & $106.67 \pm 3.04$ \\
\hline $\begin{array}{l}\text { Aggregation rate for } 30 \mathrm{~s}(\% / \mathrm{min}) \\
5 \mu \mathrm{mol} / 1\end{array}$ & $62.77 \pm 4.87$ & $100.07 \pm 2.72$ & $57.25 \pm 10.99^{*}$ & $71.60 \pm 3.38$ & $69.07 \pm 7.05$ \\
\hline $\begin{array}{l}\text { Aggregation degree (\%) } \\
10 \mu \mathrm{mol} / 1\end{array}$ & $60.73 \pm 1.45$ & $78.60 \pm 1.18$ & $53.98 \pm 3.77^{*}$ & $57.86 \pm 1.53$ & $55.93 \pm 1.85$ \\
\hline $\begin{array}{l}\text { Aggregation time }(\mathrm{s}) \\
10 \mu \mathrm{mol} / \mathrm{l}\end{array}$ & $150.83 \pm 5.25$ & $221.67 \pm 7.86$ & $153.50 \pm 9.93^{*}$ & $163.80 \pm 6.84$ & $201.00 \pm 9.97^{* *}$ \\
\hline $\begin{array}{l}\text { Aggregation rate for } 30 \mathrm{~s}(\% / \mathrm{min}) \\
10 \mu \mathrm{mol} / 1\end{array}$ & $83.05 \pm 4.46$ & $104.87 \pm 3.01$ & $72.40 \pm 5.14^{*}$ & $76.28 \pm 2.19$ & $64.47 \pm 2.81^{* *}$ \\
\hline
\end{tabular}

${ }^{*} p<0.05$ in comparison with the Con 3 -day group, ${ }^{* *} p<0.05$ in comparison with the Con 7 -day group.

aggregation, in this case, was shorter than in rats without affecting the wound defect. At the same time, on the 7 th day after modeling the wounds, the study of the effect of PBM therapy on the functional activity of rats' platelets did not show any differences in the studied parameters, except for the time to reach the maximum aggregation rate at an ADP concentration of $10 \mu \mathrm{mol} / \mathrm{l}$ (see Table 2). The shapes of the aggregation curves (single-phase reversible aggregation) did not differ in the control and experimental groups (see Figure 3).

The concentrations of ROS, bFGF, and interleukins in the blood serum of animals whose experimental wounds were exposed to PBM therapy compared to animals that did not receive PBM therapy are presented in Figure 4.

Histological studies revealed that wound healing in the animals treated with PBM therapy is characterized by a reduction in the duration of the inflammation phase compared to the control group (see Figure 5). Microscopically, this is expressed in a smaller number of polymorphonuclear leukocytes, fibrin, and tissue detritus on day 3. At the same time, fibroblasts, newly formed collagen fibers, and vessels are noted in sufficient quantities. Such signs are characteristic of the onset of the proliferation phase. In the control group, such signs are noted on day 7 (see Figure 5(b)).

Earlier onset of the remodeling phase in the experimental group of animals is evidenced by the packing of collagen fibers into bundles and their orientation parallel to the wound surface (in accordance with the mechanical load). These signs are noted on day 7 . By day 14 , the granulation tissue of the animals of the experimental group has signs of transformation into scar tissue, specifically a reduction in the number of vessels and fibroblasts, the transformation of some of the fibroblasts into fibrocytes, and compaction of bundles of collagen fibers (see Figure 5(c)). By day 28 in the experimental group, the structure of the connective tissue at the site of the wound corresponded to the histoarchitectonics of the dermis of normal skin: the thickness and location of collagen fiber bundles, the presence of hair follicles and sebaceous glands, and the absence of newly formed vessels and fibroblast proliferation. Collagen fiber bundles had a greater thickness and packing density compared to the control group. The animals of the control group showed signs of the continuation of the remodeling phase in the central sections of the wound: the presence of a significant number of fibroblasts, newly formed vessels, chaotically located, with thin collagen fibers. That is, the connective tissue was immature, and the remodeling process continued with the formation of first scar tissue and then the dermis.

In general, the results of histological examination indicate a positive effect of PBM therapy with the applied parameters on the repair processes of chronic wounds.

\section{Discussion}

The choice of radiation parameters for PBM therapy is an important task. An inappropriate choice of parameters leads 


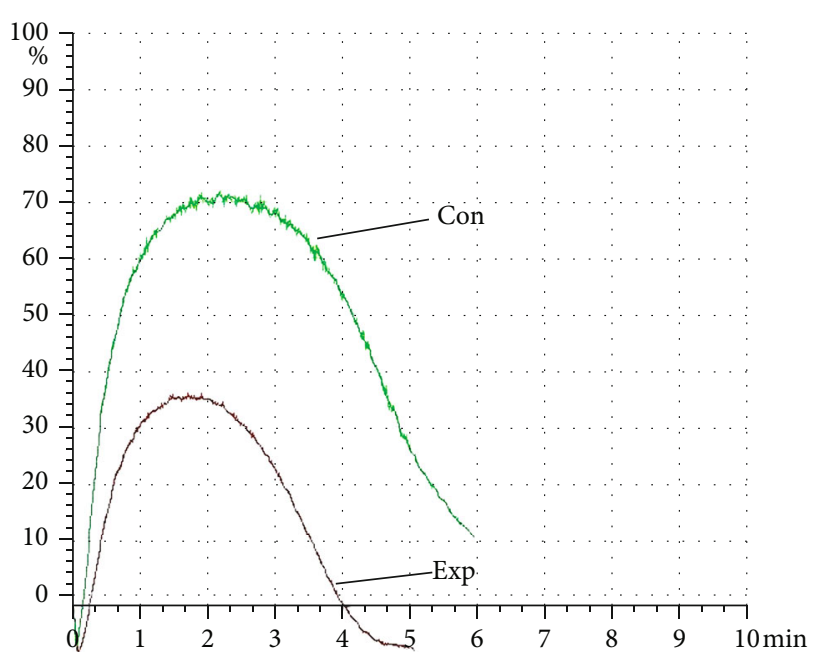

(a)

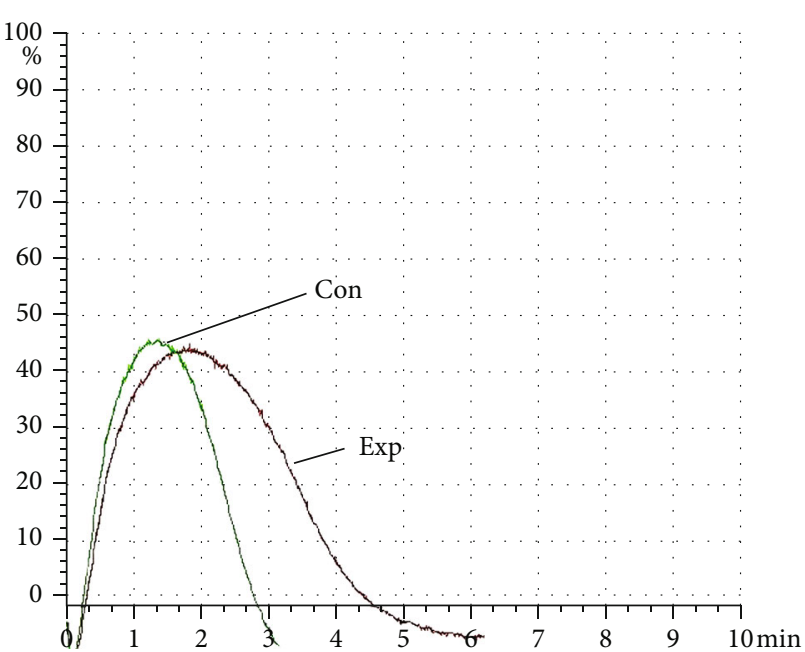

(b)

FIGURE 3: An example of the shape of the aggregation curves for animals in the control and experimental groups on day 3 (a) and day 7 (b) after surgery at an ADP concentration of $5 \mu \mathrm{mol} / \mathrm{l}$.

to unsatisfactory results or even to a negative therapeutic effect [11].

Considering that the new term "photobiomodulation therapy" is designated as "a form of light therapy...in the visible and infrared spectrum..." [12]; in previously published studies, we analyzed the effects of PBM therapy on wound regeneration processes. Wavelengths in the 600 or $800 \mathrm{~nm}$ range with daily laser exposure have been shown to be most beneficial in rodent studies, regardless of health condition or wound type [13]. The optimal therapeutic dose when using laser therapy in an open wound is from $0.5 \mathrm{~J} / \mathrm{cm}^{2}$ to $1 \mathrm{~J} / \mathrm{cm}^{2}$ [14]. Improved healing of experimental wounds in rats has been demonstrated using PBM therapy with a wavelength of $660 \mathrm{~nm}$ and energy densities of 1 and $5 \mathrm{~J} / \mathrm{cm}^{2}$ [15].

The frequency and number of PBM therapy sessions vary in the literature from one to several dozen [13]. A review by Ebrahimi et al. showed that the frequency of irradiation sessions is 3-8 times [16]. In our work, PBM therapy was applied daily for 5 days, starting the day after surgery. Literature data indicate that primary effects resulting from laser stimulation, such as an increase in the number of fibroblasts, myofibroblasts, and collagen expression, are present even after interruption of PBM therapy. Thus, after four treatment sessions carried out in the first days after wound induction, a more pronounced expression of collagen fibers was observed in rats receiving PBM therapy, compared with the control group on days 14 and 21 [17]. The results of another study also demonstrate accelerated maturation and increased collagen deposition and better architecture of final fibrous scarring at 21 days after injury, following daily application of PBM therapy during the first 7 days after wound induction [18]. In our work, we investigated the effect of PBM therapy, applied in the first 5 days after wound modeling, on the reparation processes of chronic wounds at all stages of wound healing, including the long-term effects of this method on days 14 and 28 .

Taking into account the two-phase dose-effect curve [19], we applied other radiation parameters on the wound model already used in our studies [20]: radiation power of $10 \mathrm{~mW}$ at an energy density of $1 \mathrm{~J} / \mathrm{cm}^{2}$ and a wavelength of $660 \mathrm{~nm}$

Since it is the reaction of the body as an integral system that often determines the outcome of the wound healing process (for example, in patients with diabetes), this study focused on the effect of local exposure by PBM therapy on the features of the body's response. The systemic body response, in turn, affects the local processes in the wound. Many studies have been devoted to the study of local processes directly in the wound, including by methods of immunohistochemistry. But the effect of PBM therapy on the healing of chronic wounds at the systemic level has not been adequately studied, justifying the choice of research methods utilized here.

The first response to tissue damage in the body is hemostasis. Platelets are known to be an integral part of hemostasis, but these cells also function as a physiological trigger to activate inflammation [21]. Therefore, the aggregation activity of platelets was studied in our work at the initial stages of healing. On day 3 after modeling the wound, platelet aggregation activity was increased compared to intact animals. Our results are comparable to the literature data on platelet hyperactivity in mice with impaired wound healing [22]. The platelet aggregation activity in mice was studied in pooled blood samples. We, having the opportunity to obtain a larger volume of blood in rats, investigated the aggregation activity of platelets in each animal. The use of PBM therapy in our study helped to reduce the degree, speed, and time to reach the maximum rate of aggregation for a period of 3 days after wound modeling. This is apparently due to the ability of PBM therapy to activate anti-inflammatory regulatory pathways. In our work, no differences were found in the aggregation activity of platelets between the experimental and control groups 7 days after wound simulation. Similar results were obtained in our previous study on the third day after surgery with the same wound model and similar parameters of the applied radiation (wavelength, energy density), except that the study used a radiation power of 


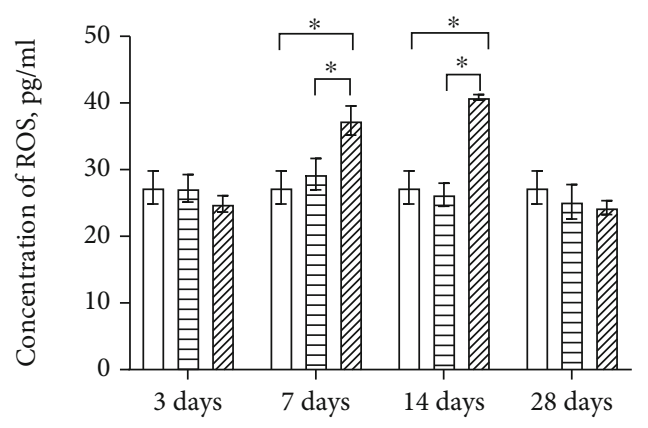

(a)

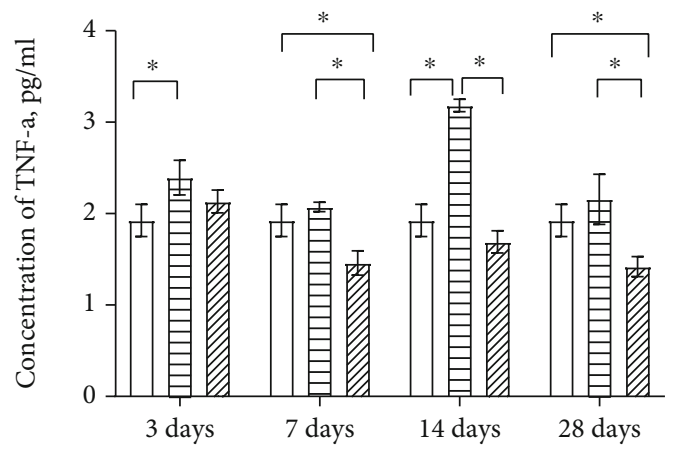

(c)

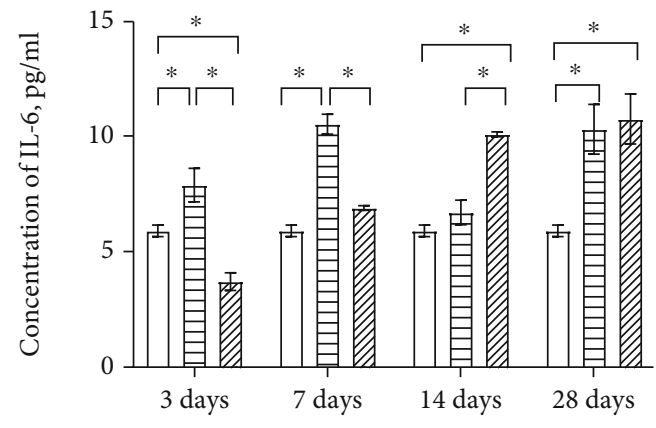

(b)

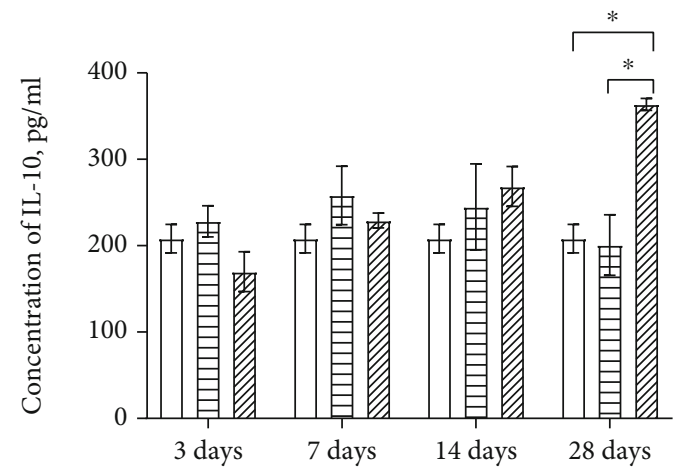

(d)

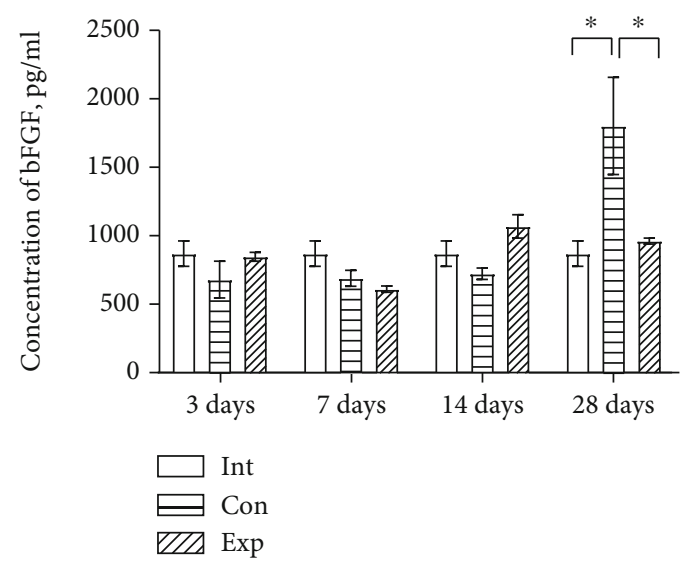

(e)

FIGURE 4: Levels of the studied indicators in the blood serum of animals: (a) ROS, (b) IL-6, (c) TNF- $\alpha$, (d) IL-10, and (e) bFGF $\left({ }^{*} p<0.05\right.$ ). Int: intact animals; Con: control group; Exp: experimental group. The error bars represent the standard error of the arithmetic mean for each indicator $(n=6)$.

$50 \mathrm{~mW}$. However, on the 7 th day after modeling the wound, an increase in platelet aggregation activity was observed [23]. This effect is apparently associated with the ability of PBM therapy to inhibit or resolve the inflammatory process [24], as well as with the pro- and anti-inflammatory functions of the platelets themselves [25].

In the regulation of platelet function, reactive oxygen species and mitochondria play a key role [26]. In our work, it was found that the action of PBM therapy on a wound defect leads to an increase in ROS levels 7 and 14 days after wound modeling. The literature provides data on an increase in the generation of ROS after PBM therapy at a wavelength of $660 \mathrm{~nm}$ [27]. In the same work, it was shown that different wavelengths have an opposite effect on the production of ROS. Many of the biological effects of PBM are attributed to the absorption of photons by chromophores located inside the mitochondria, which leads to an increase in cellular metabolic activity. Moreover, many of the mechanistic pathways for mediating the biological effects of PBM include ROS [28]. The induction of safe ROS levels observed in our study may be one of the mechanisms by which the signaling pathways that regulate cell proliferation, differentiation, and apoptosis are activated.

ROS are also important regulators of inflammation [29]. It was found that the formation of ROS originating from mitochondria induces the activation of proinflammatory 

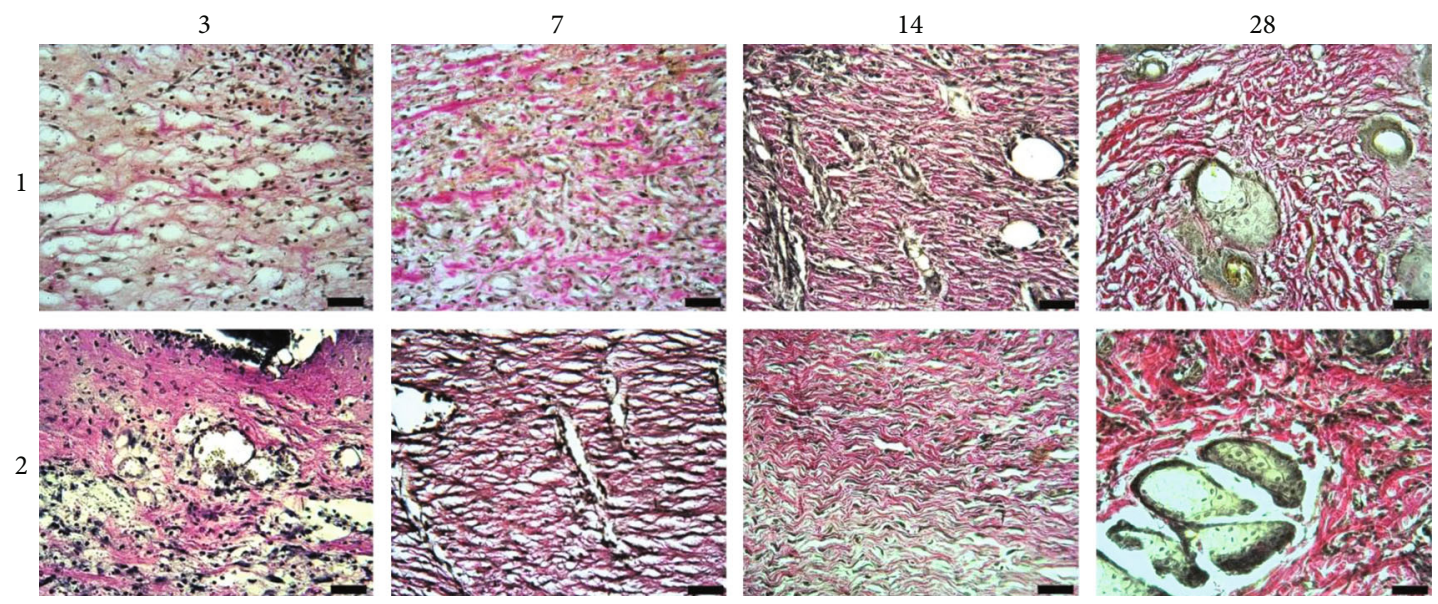

Figure 5: Animal wound sites: 1: control group ((a) on day 3, among fibrins, there are numerous polymorphonuclear leukocytes, single fibroblasts, and collagen fibers; (b) on day 7, young granulation tissue with a moderate number of polymorphonuclear leukocytes, fibroblasts and blood vessels, and chaotic collagen fibers; (c) on the 14th day, granulation tissue with signs of maturation in the form of parallel orientation and thickening of collagen fiber bundles, the presence of fibrocytes, and single vessels; (d) on the 28th day, the newly formed dermis); 2: experimental group ((a) on day 3, young granulation tissue with a moderate number of fibroblasts and polymorphonuclear leukocytes, randomly located collagen fibers, and numerous vessels; (b) on day 7 , granulation tissue with a moderate number of fibroblasts, numerous vessels, and parallel bundles of collagen fibers; (c) on the 14th day, maturing granulation tissue with parallel bundles of collagen fibers, fibrocytes, and single vessels; (d) on the 28th day, the newly formed dermis with dense bundles of collagen fibers) (H\&E, $\times 400$, scale bar $50 \mu \mathrm{m})$.

cytokines IL-1, IL-6, and TNF- $\alpha[30]$. In our study, the decrease in IL-6 levels in the experimental groups in the initial phases of healing and TNF- $\alpha$ throughout the entire wound healing process appears to be due to the antiinflammatory effect of PBM therapy at the systemic level.

Proinflammatory cytokines must perform an obligatory part of the inflammatory process because insufficient inflammation can lead to delayed healing. On the other hand, chronic wounds remain indefinitely in the inflammatory stage of wound healing [31]. To continue the healing process, each phase, upon completion of its functions, must be able to initiate the next stage (for example, resolution of inflammation shifts the process towards the proliferation phase), which is then able to turn off the previous one. Phase switching using positive and negative feedback loops is carried out by cytokines and growth factors [32]. Regulation of the levels of these intercellular mediators by PBM therapy can promote the progression of wound healing processes and prevent inhibition of progression into the next phase.

Proinflammatory cytokines are also thought to mediate cell proliferation and differentiation during wound healing. Thus, in our work, when a wound defect was exposed to low-intensity laser radiation, an increase in IL-6 levels was observed at the stage of the transition from proliferation to remodeling, which may be associated with its antiinflammatory and regenerating properties. The ability of this pleiotropic cytokine to stimulate the formation of granulation tissue, mechanisms of reepithelialization, angiogenesis, infiltration, and cell remodeling has been previously discovered [33]. There are a limited number of studies in the literature demonstrating the effect of PBM therapy on IL-6 levels in cutaneous pathology. The increase in the levels of this cytokine in the early stages of wound healing, demonstrated by Mo et al. [34], and the decrease in IL-6 concentrations after 14 and 30 days of treatment for pressure ulcers, shown by Taradaj et al. [35], do not coincide with our data. This is probably due to the difference in wound models and the studied parameters (local or systemic levels), as well as the parameters of the applied PBM therapy.

TNF- $\alpha$, like IL-6, has both proinflammatory and immunoregulatory properties. TNF- $\alpha$ provides a wide spectrum of cellular responses including regulation of inflammation, immune response, cell survival, proliferation, and apoptosis [36]. An increase in the formation of granulation tissue and a better arrangement of collagen fibers found in histological studies of wounds in the experimental group may be associated with a decrease in the levels of the cytokine TNF- $\alpha$ observed in our work [37]. Our results are comparable to the literature data, in which exposure to PBM therapy led to a decrease in the levels of the pleiotropic cytokine TNF- $\alpha[34,35,38,39]$.

The anti-inflammatory cytokine IL-10 also facilitates the transition from the inflammatory to the proliferative phase by regulating cytokine expression [40]. PBM therapy used in our work increases the levels of IL-10 in the serum of rats as inflammation decreases. This confirms the ability of PBM, in the absence of inflammation, to provide proinflammatory mediators that could help in tissue remodeling [41].

The improvement in skin regeneration of the animals of the experimental group, observed in our study, is apparently due, inter alia, to the pleiotropic effects of IL-10, including regulation of the formation of the extracellular matrix, modulation of fibroblast function, and differentiation and increased content of endothelial progenitor cells (EPC) in the wound [42]. Considering also the ability of IL-10 to counteract the deposition of collagen in scars [43], the 
regulation of levels of this cytokine by PBM therapy promotes better organization of collagen fibers, which is confirmed by our histological analysis.

Collagenogenesis is stimulated by a number of factors, including the fibroblast growth factor (FGF). bFGF is known to be a potent mitogen and chemoattractant for endothelial cells and fibroblasts. This growth factor also accelerates the formation of granulation tissue and induces reepithelialization [41]. The decrease in the maximum concentration of bFGF, observed in the blood of animals of the experimental group on day 28 after exposure to PBM therapy, apparently indicates an earlier transition of the wound healing process in the experimental group of animals to the remodeling phase. At the stage of remodeling, the number of macrophages and fibroblasts decreases due to apoptosis and regression of the new vasculature occurs, with the further transformation of granulation tissue into scar tissue [44]. The literature contains studies on the effect of PBM therapy on bFGF expression: PBM therapy stimulates the release of bFGF from human skin fibroblasts [45] and increases the concentration of this growth factor at the initial stages of wound healing [23]. The change in bFGF levels upon exposure to $\mathrm{PMB}$ therapy of $630 \mathrm{~nm}$, an energy density of $3.6 \mathrm{~J} / \mathrm{cm}^{2}$ on wounds of diabetic rats, depending on the radiation power and the timing of the wound defect, was demonstrated by Ma et al. [46].

Thus, in our study, the use of PBM therapy in the treatment of chronic wounds resulted in systemic effects. There was a decrease in platelet aggregation activity in the inflammatory phase. At all phases of the healing process, the use of this method of treatment resulted in a change in the expression of biologically active substances. The impact of PBM therapy applied locally to the wound resulted in the following changes in the central blood flow: a decrease in IL-6 levels and an increase in ROS levels in the inflammatory phase and during the transition from the inflammatory phase to the proliferation phase; an increase in IL-6 levels during the transition from the proliferation phase to the remodeling phase; a decrease in TNF- $\alpha$ levels during the transition from the inflammatory phase to the proliferation phase and further, up to the remodeling phase; and an increase in IL-10 levels and a decrease in bFGF concentration in the remodeling phase.

We believe that optimization of the activity of these biomarkers at the systemic level-as observed in our study-may be one of the mechanisms by which PBM therapy stimulates the processes of chronic wound repair. The study of systemic reactions, we hope, will make it possible in the future to develop approaches to correct violations of the repair process, supplementing the local effect of laser radiation on the wound with correction at the systemic level.

\section{Conclusions}

The results obtained in our study indicate the ability of PBM therapy to positively influence the reparation processes of chronic wounds.

The results of the histological study indicate a positive effect of PBM therapy with the applied parameters on the healing of chronic wounds, which is expressed in the acceleration of the repair processes at all phases of healing.

PBM therapy can be used to regulate wound healing processes by modulating the levels of ROS; cytokines TNF$\alpha$, IL-6, and IL-10; and bFGF.

Further research is needed to optimize radiation parameters.

\section{Data Availability}

The authors are ready to provide the main data confirming the results of the study on request.

\section{Conflicts of Interest}

The authors declare that there is no conflict of interest regarding the publication of this article.

\section{Acknowledgments}

This study was funded by the Ministry of Health of Ukraine from the state budget and was a fragment of the research work "Peculiarities of Cellular-Molecular Mechanisms of Activation of Reparative Processes in Case of Tissue Damage against the Background of a Decrease in Adaptation Reserves Characteristic of Emergency Situations" (state registration number 0120U101408).

\section{References}

[1] E. Mester, T. Spiry, B. Szende, and J. G. Tota, "Effect of laser rays on wound healing," American Journal of Surgery, vol. 122, no. 4, pp. 532-535, 1971.

[2] F. S. Cardoso, R. Á. B. Lopes Martins, and S. Gomes da Silva, “Therapeutic Potential of Photobiomodulation In Alzheimer's Disease: A Systematic Review: Photobiomodulation in Alzheimer's Disease," Journal of Lasers in Medical Sciences, vol. 11, Supplement 1, pp. S16-S22, 2020.

[3] S. S. Tomazoni, L. O. P. Costa, J. Joensen et al., "Photobiomodulation therapy is able to modulate PGE2Levels in patients with chronic non-specific low back pain: a randomized placebo-controlled trial," Lasers in Surgery and Medicine, vol. 53, no. 2, pp. 236-244, 2021.

[4] J. Ward, J. Holden, M. Grob, and M. Soldin, "Management of wounds in the community: five principles," British Journal of Community Nursing, vol. 24, Supplement 6, pp. S20-S23, 2019.

[5] L. E. Lindley, O. Stojadinovic, I. Pastar, and M. Tomic-Canic, "Biology and biomarkers for wound healing," Plastic and Reconstructive Surgery, vol. 138, Supplement 3, pp. 18S-28S, 2016.

[6] L. Deng, C. du, P. Song et al., "The role of oxidative stress and antioxidants in diabetic wound healing," Oxidative medicine and cellular longevity, vol. 2021, Article ID 8852759, 11 pages, 2021.

[7] I. Khan and P. Arany, "Biophysical approaches for oral wound healing: emphasis on photobiomodulation," Advances in Wound Care, vol. 4, no. 12, pp. 724-737, 2015.

[8] N. Houreld, "Healing effects of photobiomodulation on diabetic wounds," Applied Sciences, vol. 9, no. 23, article 5114, 2019. 
[9] D. G. Sami, H. H. Heiba, and A. Abdellatif, "Wound healing models: a systematic review of animal and non-animal models," Wound Medicine, vol. 24, no. 1, pp. 8-17, 2019.

[10] R. M. Zinatullin, T. R. Gizatullin, V. N. Pavlov et al., "Method for simulating trophic wound in experiment," in RF patent No. 2510083 C1, Federal Service for Intellectual Property (Rospatent), Ufa, Russia, 2014.

[11] M. B. Stausholm and J. M. Bjordal, "Neglect of relevant treatment recommendations in the conduct and reporting of a laser therapy knee osteoarthritis trial: letter to the editor," $B M C$ musculoskeletal disorders, vol. 22, no. 1, p. 71, 2021.

[12] J. J. Anders, R. J. Lanzafame, and P. R. Arany, "Low-level light/laser therapy versus photobiomodulation therapy," Photomedicine and Laser Surgery, vol. 33, no. 4, pp. 183-184, 2015.

[13] A. Lopez and C. Brundage, "Wound photobiomodulation treatment outcomes in animal models," Journal of Veterinary Medicine, vol. 2019, Article ID 6320515, 9 pages, 2019.

[14] J. Tunér and L. Hode, Laser Therapy - Clinical Practice and Scientific Background, Prima Books AB, Grangesberg, Sweden, 2002.

[15] R. Suzuki and K. Takakuda, "Wound healing efficacy of a 660$\mathrm{nm}$ diode laser in a rat incisional wound model," Lasers in Medical Science, vol. 31, no. 8, pp. 1683-1689, 2016.

[16] P. Ebrahimi, M. Hadilou, F. Naserneysari et al., "Effect of photobiomodulation in secondary intention gingival wound healing - a systematic review and meta-analysis," BMC Oral Health, vol. 21, no. 1, 2021.

[17] T. Fortuna, A. C. Gonzalez, M. F. Sá, Z. . A. Andrade, S. R. A. Reis, and A. R. A. P. Medrado, "Effect of $670 \mathrm{~nm}$ laser photobiomodulation on vascular density and fibroplasia in late stages of tissue repair," International Wound Journal, vol. 15, no. 2, pp. 274-282, 2018.

[18] J. L. S. Cunha, F. M. A. de Carvalho, R. N. Pereira Filho, M. A. G. Ribeiro, and R. L. C. de Albuquerque-Júnior, "Effects of different protocols of low-level laser therapy on collagen deposition in wound healing," Brazilian Dental Journal, vol. 30, no. 4, pp. 317-324, 2019.

[19] Y. Y. Huang, A. C. Chen, J. D. Carroll, and M. R. Hamblin, "Biphasic dose response in low level light therapy," DoseResponse, vol. 7, no. 4, pp. 358-383, 2009.

[20] S. B. Pavlov, N. M. Babenko, M. V. Kumetchko, O. B. Litvinova, N. G. Semko, and R. N. Mikhaylusov, "The influence of photobiomodulation therapy on chronic wound healing," Romanian reports in physics, vol. 72, article 609, 2020.

[21] W. R. Parrish and B. Roides, "Physiology of blood components in wound healing: an appreciation of cellular co-operativity in platelet rich plasma action," Journal of Exercise, Sports \& Orthopedics, vol. 4, no. 2, pp. 1-14, 2017.

[22] S. Dhall, Z. A. Karim, F. T. Khasawneh, and M. MartinsGreen, "Platelet hyperactivity inTNFSF14/LIGHTKnockout mouse model of impaired healing," Advances in Wound Care, vol. 5, no. 10, pp. 421-431, 2016.

[23] N. Babenko and S. Pavlov, "Effects of low-level laser therapy on reactive oxygen species, platelet aggregation activity, and the expression of growth factors in the process of regeneration of chronic wounds," Annals of the Romanian Society for Cell Biology, vol. 25, no. 4, pp. 2226-2234, 2021.

[24] R. A. B. Lopes-Martins, P. S. Leonardo, J. M. Bjordal, and R. L. Marcos, "Photobiomodulation: inhibition or resolution of the inflammatory process?," Photobiomodulation, photomedicine, and laser surgery, vol. 38, no. 8, pp. 453-454, 2020.

[25] A. Margraf and A. Zarbock, "Platelets in inflammation and resolution," Journal of Immunology (Baltimore, Md. : 1950), vol. 203, no. 9, pp. 2357-2367, 2019.

[26] E. Masselli, G. Pozzi, M. Vaccarezza et al., "ROS in platelet biology: functional aspects and methodological insights," International journal of molecular sciences, vol. 21, no. 14, article ???, 2020.

[27] K. Rupel, L. Zupin, A. Colliva et al., "Photobiomodulation at multiple wavelengths differentially modulates oxidative stress in vitro and in vivo," Oxidative medicine and cellular longevity, vol. 2018, Article ID 6510159, 2018.

[28] M. R. Hamblin, "Mechanisms and mitochondrial redox signaling in photobiomodulation," Photochemistry and Photobiology, vol. 94, no. 2, pp. 199-212, 2018.

[29] R. Mehling, J. Schwenck, C. Lemberg et al., "Immunomodulatory role of reactive oxygen species and nitrogen species during $\mathrm{T}$ cell-driven neutrophil-enriched acute and chronic cutaneous delayed-type hypersensitivity reactions," Theranostics, vol. 11, no. 2, pp. 470-490, 2021.

[30] E. Naik and V. M. Dixit, "Mitochondrial reactive oxygen species drive proinflammatory cytokine production," The Journal of Experimental Medicine, vol. 208, no. 3, pp. 417420, 2011.

[31] P. Krzyszczyk, R. Schloss, A. Palmer, and F. Berthiaume, "The role of macrophages in acute and chronic wound healing and interventions to promote pro-wound healing phenotypes," Frontiers in physiology, vol. 9, article 419, 2018.

[32] N. B. Menke, K. R. Ward, T. M. Witten, D. G. Bonchev, and R. F. Diegelmann, "Impaired wound healing," Clinics in Dermatology, vol. 25, no. 1, pp. 19-25, 2007.

[33] Y. Feng, A. J. Sanders, L. D. Morgan, K. G. Harding, and W. G. Jiang, "Potential roles of suppressor of cytokine signaling in wound healing," Regenerative Medicine, vol. 11, no. 2, pp. 193-209, 2016.

[34] S. Mo, P. S. Chung, and J. C. Ahn, “630 nm-OLED accelerates wound healing in mice via regulation of cytokine release and genes expression of growth factors," Current Optics and Photonics, vol. 3, no. 6, pp. 485-495, 2019.

[35] J. Taradaj, B. Shay, R. Dymarek et al., "Effect of laser therapy on expression of angio- and fibrogenic factors, and cytokine concentrations during the healing process of human pressure ulcers," International Journal of Medical Sciences, vol. 15, no. 11, pp. 1105-1112, 2018.

[36] N. Petryk and O. Shevchenko, "Mesenchymal stem cells antiinflammatory activity in rats: proinflammatory cytokines," Journal of Inflammation Research, vol. Volume 13, pp. 293 $301,2020$.

[37] M. B. Serra, W. A. Barroso, N. N. . Silva et al., "From inflammation to current and alternative therapies involved in wound healing," International journal of inflammation, vol. 2017, Article ID 3406215, 17 pages, 2017.

[38] G. K. Keshri, A. Gupta, A. Yadav, S. K. Sharma, and S. B. Singh, "Photobiomodulation with pulsed and continuous wave nearinfrared laser (810 nm, Al-Ga-As) augments dermal wound healing in immunosuppressed rats," PLoS One, vol. 11, no. 11, article e0166705, 2016.

[39] A. N. Otterço, A. L. Andrade, P. Brassolatti, K. N. Z. Pinto, H. S. S. Araújo, and N. A. Parizotto, "Photobiomodulation mechanisms in the kinetics of the wound healing process in 
rats," Journal of Photochemistry and Photobiology B: Biology, vol. 183, pp. 22-29, 2018.

[40] S. Willenborg and S. A. Eming, "Macrophages - sensors and effectors coordinating skin damage and repair," Journal of the German Society of Dermatology: JDDG, vol. 12, no. 3, pp. 214-223, 2014.

[41] L. F. de Freitas and M. R. Hamblin, "Proposed mechanisms of photobiomodulation or low-level light therapy," IEEE journal of selected topics in quantum electronics: a publication of the IEEE Lasers and Electro-optics Society, vol. 22, no. 3, article 7000417, 2016.

[42] A. King, S. Balaji, L. D. Le, T. M. Crombleholme, and S. G. Keswani, "Regenerative wound healing: the role of interleukin-10," Advances in Wound Care, vol. 3, no. 4, pp. 315323, 2014.

[43] K. L. Singampalli, S. Balaji, X. Wang et al., "The role of an IL10/hyaluronan axis in dermal wound healing," Frontiers in cell and developmental biology, vol. 8, article 636, 2020.

[44] M. Rodrigues, N. Kosaric, C. A. Bonham, and G. C. Gurtner, "Wound healing: a cellular perspective," Physiological Reviews, vol. 99, no. 1, pp. 665-706, 2019.

[45] M. Esmaeelinejad and M. Bayat, "Effect of low-level laser therapy on the release of interleukin- 6 and basic fibroblast growth factor from cultured human skin fibroblasts in normal and high glucose mediums," Journal of cosmetic and laser therapy : official publication of the European Society for Laser Dermatology, vol. 15, no. 6, pp. 310-317, 2013.

[46] H. Ma, Y. Li, H. Chen, M. Kang, and T. C. Y. Liu, "Effects of low-intensity laser irradiation on wound healing in diabetic rats," International Journal of Photoenergy, vol. 2012, Article ID 838496, ll pages, 2012. 